\title{
DETERMINAÇÃO DE ÁREAS DE ENDEMISMO DE CORAIS ESCLERACTÍNEOS E HIDROCORAIS NO ATLÂNTICO SUL OCIDENTAL
}

\author{
Samira da Conceição Sabadini ${ }^{1}$
}

\section{Resumo}

Os recifes de corais são considerados o ecossistema marinho de maior produtividade, sendo os recifes brasileiros os mais importantes do Oceano Atlântico Sul Ocidental. A costa brasileira apresenta pouca biodiversidade de corais hermatípicos e considerável quantidade de espécies endêmicas. Este trabalho teve como objetivo geral determinar as áreas de endemismo de corais escleractíneos e hidrocorais nos recifes brasileiros e, mais especificamente, definir as áreas que demandam atenção especial para a proteção das espécies endêmicas no Brasil. O estudo foi desenvolvido para os recifes do Parcel de Manuel Luis, Maracajaú, Atol das Rocas, Arquipélago de Fernando de Noronha, Tamandaré, Maragogi, costa do Estado da Bahia e região de desaparecimento de corais hermatípicos. Os registros de espécies ao longo da costa foram obtidos da literatura. Foi utilizada a metodologia GeographicalInterpolation of Endemism (GIE) desenvolvida por Oliveira et al (2015) e, então, gerado o mapa de áreas de endemismo a partir da função de interpolação Espacial Kernel. A metodologia foi adequada porque o mapa permitiu a compreensão das áreas de sindemia de corais hermatípicos em duas escalas espaciais.

Palavras-chave:Recifes de corais, endemismo, Interpolação Espacial Kernel.

\begin{abstract}
Coral reefs are consideredthe marine ecosystem of hightestproductivity, and theBrazilianreefsthemostimportant in theSouthwesternAtlantic Ocean. The Braziliancoasthaslowbiodiversityhermatypiccorals and considerableamount of endemicspecies. Thisworkaimedto determine theareas of endemism of scleractiniancorals and hydrocorals in Brazilianreefs and, more specifically define theareasthatrequirespecialattentiontoprotecttheendemicspecies in Brazil. The studywasconducted for thereefs of Parcel de Manuel Luis, Maracajaú, Rocas Atoll, Fernando de Noronha Archipelago, Tamandaré, Maragogi, Cost of the State of Bahia and thedisappearance of coral hermatypic region. Records of speciesalongthecoastwereobtainedfromtheliterature. The methodologyusedwasGeographicalInterpolation of endemism (GIE) developedby Oliveira et al (2015) and thenwasgeneratedthemap of endemicareasfromtheSpatialKernelinterpolationfunction. The methodologywasappropriatebecausethemapallowedtheunderstanding of theareas of syndemic of hermatypiccoralsontwospatialscales.
\end{abstract}

Keywords:Coral Reefs, endemism, SpatialInterpolationKernel.

\footnotetext{
1 Mestranda do Programa de Pós-Graduação em Análise e Modelagem de Sistemas Ambientais IGC-UFMG samirasabadini@yahoo.com.br
} 


\section{1- INTRODUÇÃO}

Os recifes de corais são considerados o ecossistema marinho de maior produtividade, sendo os recifes brasileiros os mais importantes do Oceano Atlântico Sul Ocidental (KIKUCHI et al, 2003, p. 1; LEÃO et al, 2010, p. 224). A costa brasileira apresenta pouca biodiversidade de corais e considerável quantidade de espécies endêmicas como a Mussismilia brasiliensis, Mussismilia hispida, Mussismiliaharttii, Siderastreastellata, Favia gravida, Favialeptophylla, Milleporabraziliensis e Milleporanitida (LEÃO et al, 2003, p. 14-17).

As espécies Mussismilia brasiliensis, Mussismilia hispida, Mussismiliaharttii e Favialeptophylla constituem relíquias do período terciário que foram preservadas nos montes submarinos durante a regressão marinha do Pleistoceno. Já a Siderastreastellata e a Favia gravida apresentam similaridades com espécies caribenhas (LEÃO et al, 2003, p. 14). Essas espécies estão adaptadas à condição de considerável turbidez das águas costeiras brasileiras, pois a presença de grandes coralitos favoreceu sua sobrevivência nas águas da costa brasileira (LEÃO et al, 2003, p. 17).

Uma forma de determinar as áreas de endemismo das espécies consideradas é através da aplicação do método GeographicalInterpolation of Endemism (GIE) que tem como base a função de Interpolação Espacial Kernel (OLIVEIRA et al., 2015, p.1). Este método consiste na definição de áreas de influência (raio) para o centroide de cada espécie e de uma função Gaussiana de decaimento de intensidade a partir dos centroides. A sobreposição de espécies endêmicas (sindemia) dentro das áreas de influência é representada em um mapa de densidades proporcionais à quantidade de espécies sobrepostas (OLIVEIRA et al., 2015, p.3). Diante da importância da conservação das áreas recifais na costa brasileira, torna-se fundamental o estudo das áreas de endemismo e possíveis lacunas de conservação.

Segundo o Ministério do Meio Ambiente (acesso em 03 de nov. de 2015), no Brasil, as unidades de conservação de áreas recifais são: Parque Estadual Marinho do Parcel do Manuel Luis (MA), Reserva Biológica do Atol das Rocas (RN), Parque Nacional Marinho de Fernando de Noronha (PE), Área de Proteção Ambiental de Fernando de Noronha - Rocas - São Pedro e São Paulo, Área de Proteção Ambiental Estadual dos Recifes de Corais, Parque Estadual Marinho da Areia Vermelha, Área de Proteção Ambiental Costa dos Corais (PE/AL), Área de Proteção Ambiental do Litoral Norte (BA), Área de Proteção Ambiental da Baía de Todos os Santos (BA), Área de Proteção Ambiental Municipal Recifes de Pinaúnas (BA), Área de Proteção Ambiental Tinharé- Boipeba (BA), Área de Proteção Ambiental da Baía de Camamu (BA), Parque Municipal Marinho da Coroa Alta (BA), Parque Municipal Marinho do Recife de Fora (BA), Reserva 
Extrativista Marinha de Corumbau (BA), Parque Municipal Marinho do Recife de Areia (BA), Parque Nacional Marinho de Abrolhos (BA) e Área de Proteção Ambiental Ponta da Baleia/Abrolhos.

No entanto, apenas $1,57 \%$ do território marinho brasileiro é protegido por unidades de conservação, sendo a maior parte (1,43\%) de uso sustentável (MMA, acesso em 03 nov. 2015). $\mathrm{Na}$ região de Abrolhos alguns recifes de borda de plataforma encontram-se fora dos limites de áreas protegidas, o que constitui uma lacuna de conservação (MMA, 2012, p. 14). Nesse sentido, o Instituto Chico Mendes de Conservação da Biodiversidade (ICMBio) propôs a criação de unidades de conservação e a ampliação do Parque Nacional Marinho de Abrolhos para aumentar a proteção do ecossistema recifal de maior biodiversidade no Atlântico Sul (MMA, 2012, p.3).

Portanto, é evidente que as unidades de conservação marinhas no Brasil são insuficientes para proteger os recifes de corais dos efeitos de recorrentes fenômenos naturais e ameaças antrópicas.

Este trabalho tem como objetivo geral determinar as áreas de endemismo de corais hermatípicos na costa brasileira e, mais especificamente, definir as áreas que demandam atenção especial para a proteção das espécies endêmicas no Brasil.

\section{2- ÁREA DE ESTUDO}

Este trabalho abrange recifes e áreas da costa brasileira onde há registro das espécies endêmicas de corais escleractíneos e hidrocorais no Atlântico Sul Ocidental. As áreas recifais estudadas compreendem o Parcel de Manuel Luís, Atol das Rocas, Arquipélago de Fernando de Noronha, Maracajaú, Tamandaré, Maragogi e a costa da Bahia. As áreas que não constituem recifes propriamente ditos, mas que possuem registros de ocorrências das espécies endêmicas, são: Ilha de Trindade, Rio de Janeiro, Espírito Santo, São Paulo e Santa Catarina (Figura 1). Nos Apêndices (A, B, C e D) são apresentados os locais de ocorrências de cada espécie endêmica de corais e hidrocorais na costa brasileira. 


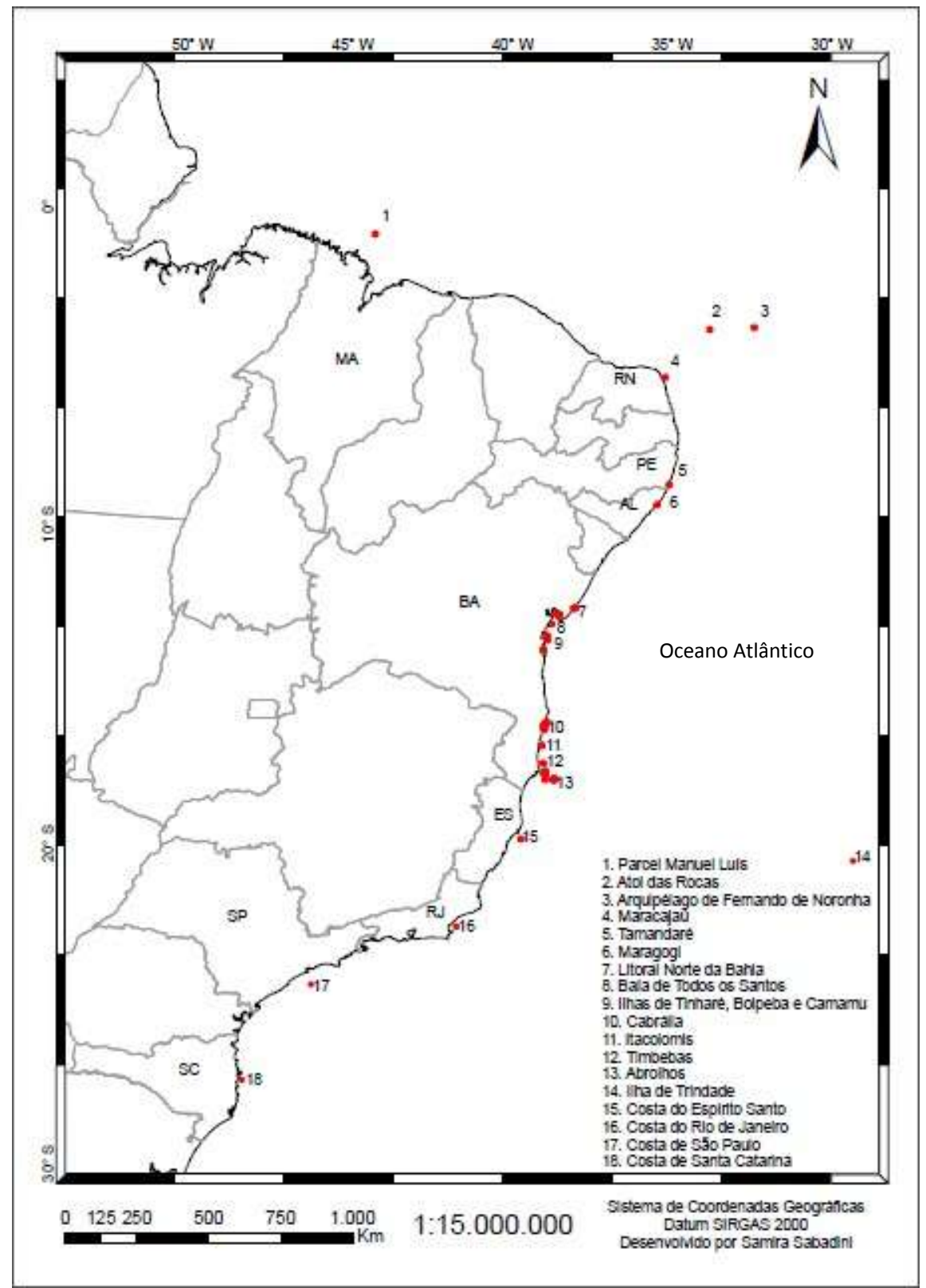

Figura 1: Localização dos registros de espécies de corais escleractíneos e hidrocorais endêmicos da costa brasileira.

As áreas recifais da Bahia (Figura 2) distribuem-se entre as latitudes de 12 a $18^{\circ} \mathrm{S}$, sendo as mais ricas do Atlântico Sul Ocidental (KIKUCHI ET AL, 2010, p. 1). Abrangem 18 espécies escleractíneas e 4 hidrocorais, das quais 8 são endêmicas no Atlântico Sul Ocidental (LEÃO et al., 2010, p.225). 


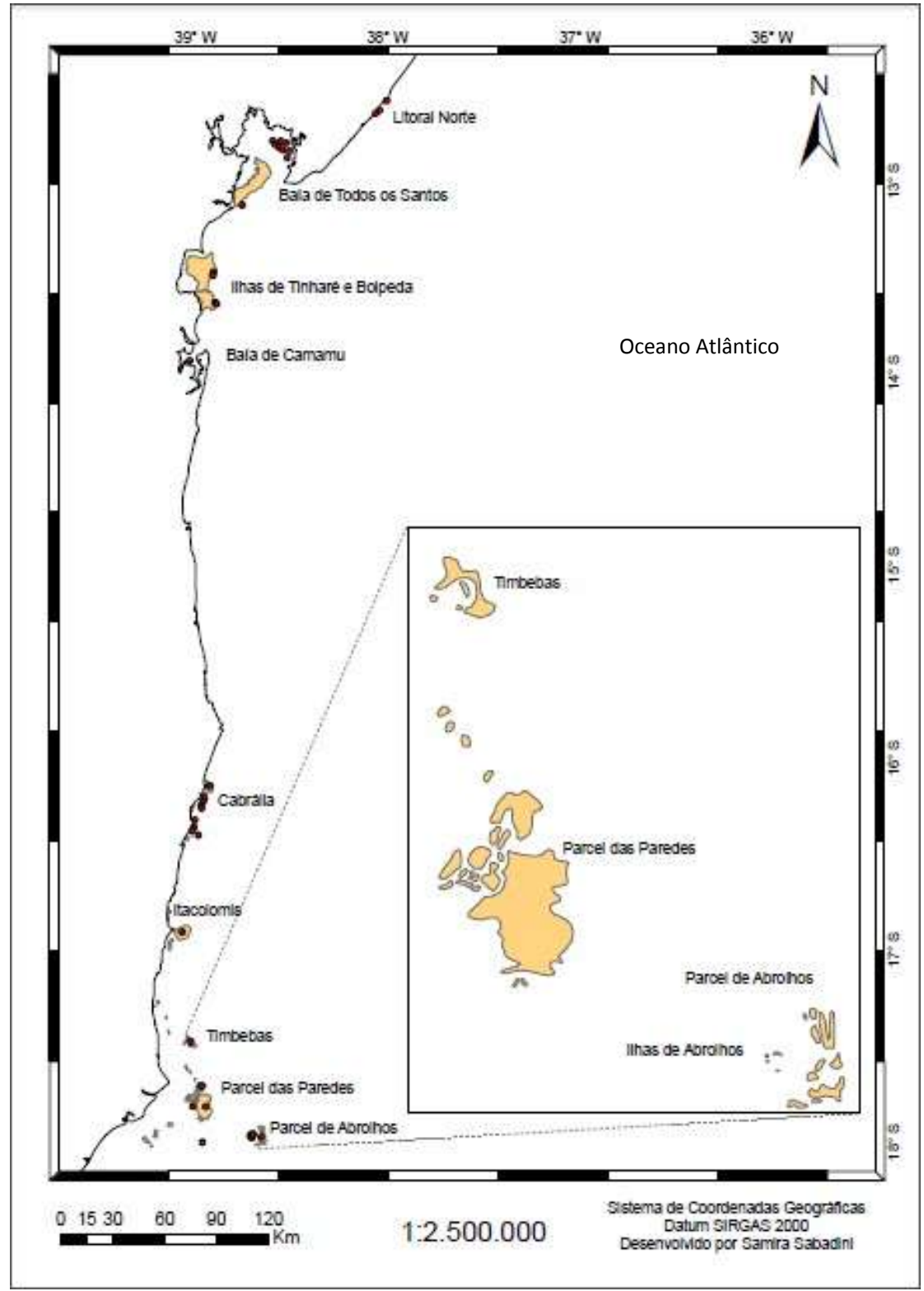

Figura 2: Áreas recifais do Estado da Bahia: Litoral norte, Baía de Todos os Santos, Ilhas de Tinharé e Boipeda e Baía de Camamu, Cabrália e Porto Seguro, Itacolomis e Complexo Recifal de Abrolhos (em destaque).

\section{3- BASES CARTOGRÁFICAS}

As bases cartográficas de limites das áreas recifais e da linha de costa utilizadas neste estudo foram disponibilizadas pelo Serviço Geológico do Brasil (CPRM). A base cartográfica referente às unidades de conservação marinha de proteção integral e uso sustentável foi disponibilizada pelo Instituto Brasileiro de Geografia e Estatística (IBGE). A localização das ocorrências de espécies de corais escleractíneos e hidrocorais (Tabela 1) foram obtidas da literatura e transformadas em shapefile no software ArcGis 10.1. 
TABELA 1. Espécies endêmicas de corais escleractíneos e hidrocorais registradas na costa brasileira. Baseado em Castro (2000), Santos et al (2007), Echeverría et al (1997), Cavalcante et al (2014), Pinheiro (2006), Kikuchi et al (2010) e Laborel (1969).

\begin{tabular}{|c|c|c|c|c|c|c|c|c|}
\hline Local/Espécie & $\begin{array}{l}\text { Sid } \\
\text { erastel } \\
\text { strlat } \\
\text { ea a }\end{array}$ & $\begin{array}{c}\text { Fagra } \\
\text { via }{ }^{\text {vid }} \\
\quad a\end{array}$ & $\begin{array}{c}\text { lep } \\
\text { Fatop } \\
\text { viahyl } \\
\text { la }\end{array}$ & $\begin{array}{l}\text { Mubra } \\
\text { ssis zili } \\
\text { mil }_{\text {ens }} \\
\text { ia is }\end{array}$ & $\begin{array}{l}\text { Mu } \\
\text { ssis } \\
\text { mil }_{\text {pid }} \\
{ }_{\text {ia }}{ }_{a}\end{array}$ & $\begin{array}{l}\text { Mu } \\
\text { ssis } \\
\text { milhar } \\
\text { ia ttii }\end{array}$ & $\begin{array}{c}\text { Mibra } \\
\text { lepzili } \\
\text { ordens } \\
\text { is }\end{array}$ & $\begin{array}{l}\text { Mil } \\
\text { lep }_{\text {niti }} \\
\text { ora }_{d a}\end{array}$ \\
\hline
\end{tabular}

\begin{tabular}{lllllll}
\hline $\begin{array}{l}\text { Parcel Manuel } \\
\text { Luis }\end{array}$ & $\mathrm{X}$ & $\mathrm{X}$ & $\mathrm{X}$ & $\mathrm{X}$ & & $\mathrm{X}$ \\
Maracajaú & $\mathrm{X}$ & $\mathrm{X}$ & & & $\mathrm{X}$ & $\mathrm{X}$ \\
Atol das Rocas & $\mathrm{X}$ & $\mathrm{X}$ & & $\mathrm{X}$ & & $\mathrm{X}$ \\
Fernando & $\mathrm{X}$ & $\mathrm{X}$ & & $\mathrm{X}$ & $\mathrm{X}$ &
\end{tabular}

Noronha

$\begin{array}{lllllll}\text { Tamandaré } & \mathrm{X} & \mathrm{X} & & \mathrm{X} & \mathrm{X} & \mathrm{X} \\ \text { Maragogi } & \mathrm{X} & \mathrm{X} & & \mathrm{X} & \mathrm{X} & \mathrm{X} \\ \text { Praia do Forte }^{1} & \mathrm{X} & \mathrm{X} & \mathrm{X} & \mathrm{X} & & \\ \text { Itacimirim }^{1} & \mathrm{X} & \mathrm{X} & \mathrm{X} & \mathrm{X} & \mathrm{X} & \\ \text { Gaurajuba }^{1} & \mathrm{X} & \mathrm{X} & \mathrm{X} & \mathrm{X} & & \\ \text { Mangueira }^{2} & \mathrm{X} & & & \mathrm{X} & & \\ \text { Gaituba }^{2} & \mathrm{X} & & & & & \\ \text { Pedra }_{\text {Me }}^{\text {do }} & \mathrm{X} & & & \mathrm{X} & & \end{array}$

Dentão $^{2}$

\begin{tabular}{|c|c|c|c|c|c|c|}
\hline Cardinal $^{2}$ & $\mathrm{X}$ & & & $\mathrm{X}$ & & \\
\hline Poste $1^{2}$ & $\mathrm{X}$ & & & $\mathrm{X}$ & & \\
\hline Poste $4^{2}$ & $\mathrm{X}$ & & & $\mathrm{X}$ & & \\
\hline Poste $5^{2}$ & $\mathrm{X}$ & & & $\mathrm{X}$ & & \\
\hline Poste $6^{2}$ & $\mathrm{X}$ & $\mathrm{X}$ & & $\mathrm{X}$ & $\mathrm{X}$ & \\
\hline Caramuanas $^{2}$ & $\mathrm{X}$ & & $\mathrm{X}$ & $\mathrm{X}$ & & \\
\hline $\begin{array}{ll}\text { Praia } & \text { do } \\
\text { Quadro }^{3} & \end{array}$ & $\mathrm{X}$ & $\mathrm{X}$ & $\mathrm{X}$ & $\mathrm{X}$ & & \\
\hline Garapuá3 & $\mathrm{X}$ & & $\mathrm{X}$ & $\mathrm{X}$ & $\mathrm{X}$ & \\
\hline Morerá ${ }^{3}$ & $\mathrm{X}$ & $\mathrm{X}$ & $\mathrm{X}$ & $\mathrm{X}$ & $\mathrm{X}$ & \\
\hline Bainema $^{3}$ & $\mathrm{X}$ & & $\mathrm{X}$ & $\mathrm{X}$ & & \\
\hline Camamu $^{3}$ & $\mathrm{X}$ & $\mathrm{X}$ & $\mathrm{X}$ & $\mathrm{X}$ & $\mathrm{X}$ & $\mathrm{X}$ \\
\hline Aragipe 4 & $\mathrm{X}$ & $\mathrm{X}$ & $\mathrm{X}$ & $\mathrm{X}$ & $\mathrm{X}$ & $\mathrm{X}$ \\
\hline $\begin{array}{ll}\text { Coroa } & \text { Alta } \\
\text { Norte }^{4} & \end{array}$ & $\mathrm{X}$ & $\mathrm{X}$ & $\mathrm{X}$ & $\mathrm{X}$ & $\mathrm{X}$ & $\mathrm{X}$ \\
\hline Coroa & $\mathrm{X}$ & $\mathrm{X}$ & & $\mathrm{X}$ & $\mathrm{X}$ & $\mathrm{X}$ \\
\hline
\end{tabular}

\begin{tabular}{lllllll} 
Sul & & & & & \\
Itassepanema $^{4}$ & $\mathrm{X}$ & $\mathrm{X}$ & $\mathrm{X}$ & $\mathrm{X}$ & $\mathrm{X}$ & $\mathrm{X}$ \\
Alagadas $^{4}$ & $\mathrm{X}$ & $\mathrm{X}$ & $\mathrm{X}$ & $\mathrm{X}$ & $\mathrm{X}$ & $\mathrm{X}$ \\
Naufrágio $^{4}$ & $\mathrm{X}$ & $\mathrm{X}$ & $\mathrm{X}$ & $\mathrm{X}$ & $\mathrm{X}$ & \\
\hline Coroa & $\mathrm{X}$ & $\mathrm{X}$ & & & & $\mathrm{X}$
\end{tabular}

Vermelha ${ }^{4}$

Cadernos do Leste

Artigos Cientificos

Belo Horizonte, Jan.-Dez. Vol.16, n¹6, 2016 


\begin{tabular}{|c|c|c|c|c|c|c|c|}
\hline Ponta Grande 4 & $\mathrm{X}$ & $\mathrm{X}$ & & & & & $\mathrm{X}$ \\
\hline Recife de fora ${ }^{4}$ & $\mathrm{X}$ & $\mathrm{X}$ & & $\mathrm{X}$ & $\mathrm{X}$ & $\mathrm{X}$ & \\
\hline Itacolomis 5 & $\mathrm{X}$ & $\mathrm{X}$ & $\mathrm{X}$ & $\mathrm{X}$ & $\mathrm{X}$ & $\mathrm{X}$ & $\mathrm{X}$ \\
\hline Leste $^{6}$ & $\mathrm{X}$ & $\mathrm{X}$ & $\mathrm{X}$ & $\mathrm{X}$ & $\mathrm{X}$ & $\mathrm{X}$ & $\mathrm{X}$ \\
\hline Lixa $^{6}$ & $\mathrm{X}$ & $\mathrm{X}$ & $\mathrm{X}$ & $\mathrm{X}$ & $\mathrm{X}$ & $\mathrm{X}$ & $\mathrm{X}$ \\
\hline Paredes $^{6}$ & $\mathrm{X}$ & $\mathrm{X}$ & $\mathrm{X}$ & $\mathrm{X}$ & $\mathrm{X}$ & $\mathrm{X}$ & $\mathrm{X}$ \\
\hline Timbebas ${ }^{6}$ & $\mathrm{X}$ & $\mathrm{X}$ & $\mathrm{X}$ & $\mathrm{X}$ & $\mathrm{X}$ & $\mathrm{X}$ & \\
\hline Popa Verde 6 & $\mathrm{X}$ & $\mathrm{X}$ & $\mathrm{X}$ & $\mathrm{X}$ & $\mathrm{X}$ & $\mathrm{X}$ & \\
\hline $\begin{array}{l}\text { Santa Bárbara } \\
\text { Sul }^{6}\end{array}$ & $\mathrm{X}$ & $\mathrm{X}$ & $\mathrm{X}$ & $\mathrm{X}$ & $\mathrm{X}$ & $\mathrm{X}$ & \\
\hline $\begin{array}{l}\text { Santa Bárbara } \\
\text { Oeste }^{6}\end{array}$ & $\mathrm{X}$ & $\mathrm{X}$ & & $\mathrm{X}$ & $\mathrm{X}$ & $\mathrm{X}$ & \\
\hline
\end{tabular}

\begin{tabular}{|c|c|c|c|c|c|c|}
\hline $\begin{array}{l}\text { Santa Bárbara } \\
\text { Norte }^{6}\end{array}$ & $\mathrm{X}$ & $\mathrm{X}$ & $\mathrm{X}$ & $\mathrm{X}$ & $\mathrm{X}$ & $\mathrm{X}$ \\
\hline Sueste $^{6}$ & $\mathrm{X}$ & $\mathrm{X}$ & & $\mathrm{X}$ & $\mathrm{X}$ & $\mathrm{X}$ \\
\hline Siriba $^{6}$ & $\mathrm{X}$ & $\mathrm{X}$ & $\mathrm{X}$ & $\mathrm{X}$ & $X$ & $\mathrm{X}$ \\
\hline Redonda ${ }^{6}$ & $\mathrm{X}$ & $\mathrm{X}$ & & $\mathrm{X}$ & $X$ & X \\
\hline Guarita $^{6}$ & $\mathrm{X}$ & $\mathrm{X}$ & $\mathrm{X}$ & $\mathrm{X}$ & $X$ & $\mathrm{X}$ \\
\hline Parcel & $\mathrm{X}$ & $\mathrm{X}$ & X & $\mathrm{X}$ & $\mathrm{X}$ & X \\
\hline
\end{tabular}

Abrolhos ${ }^{6}$

$\begin{array}{lllll}\text { Espírito Santo } & \mathrm{X} & \mathrm{X} & \mathrm{X} & \mathrm{X} \\ \mathrm{X}\end{array}$

Ilha de $\mathrm{X}$

Trindade

\begin{tabular}{lll} 
Rio de Janeiro & $\mathrm{X}$ & $\mathrm{X}$ \\
São Paulo & & $\mathrm{X}$ \\
Santa Catarina & $\mathrm{X}$ \\
\hline
\end{tabular}

1: Litoral norte da Bahia; 2: Baía de Todos os Santos; 3: Tinharé, Boipeba e Camamu; 4: Cabrália e Porto Seguro; 5: Itacolomis; 6: Abrolhos.

\section{4- INTERPOLAÇÃO KERNEL}

Para realizar a interpolação espacial Kernel foi utilizado o software ArcGis $10.1 \mathrm{com}$ a ferramenta GIE desenvolvida por Oliveira et al (2015). O método utilizado baseia-se nas seguintes etapas (Figura 3): a) Estimação do centroide a partir da média aritmética das latitudes e longitudes de registros das ocorrências de espécies; b) Definição do raio a partir da maior distância entre o centroide e as ocorrências de cada espécie; c) Agrupamentos de espécies em classes de acordo com o tamanho do raio definido para cada espécie; d) Sobreposição de áreas de influência de acordo com uma função Gaussiana que diminui a intensidade ao se afastar do centroide; e - f) Delimitação das áreas de endemismo de espécies. 


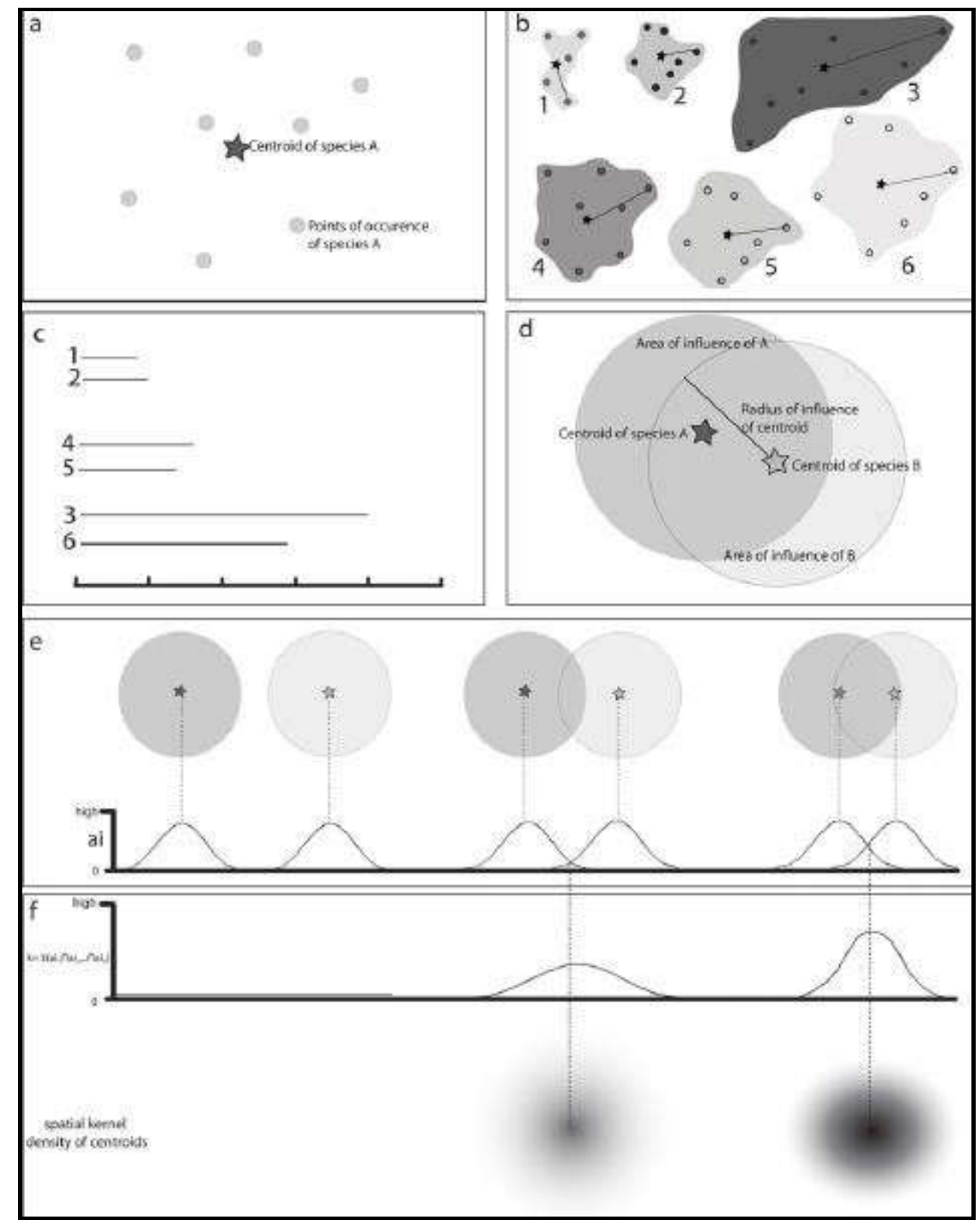

Figura 3: Etapas do método GIE.

Fonte: Oliveira et al (2015).

\section{5- RESULTADOS E DISCUSSÃO}

O método GIE foi utilizado em duas escalas espaciais: Nacional e regional. Na escala nacional, foram identificadas duas áreas de endemismo na costa brasileira. Na Figura 4 são apresentadas as áreas de endemismo de espécies de corais hermatípicos no Oceano Attântico Sul Ocidental. 


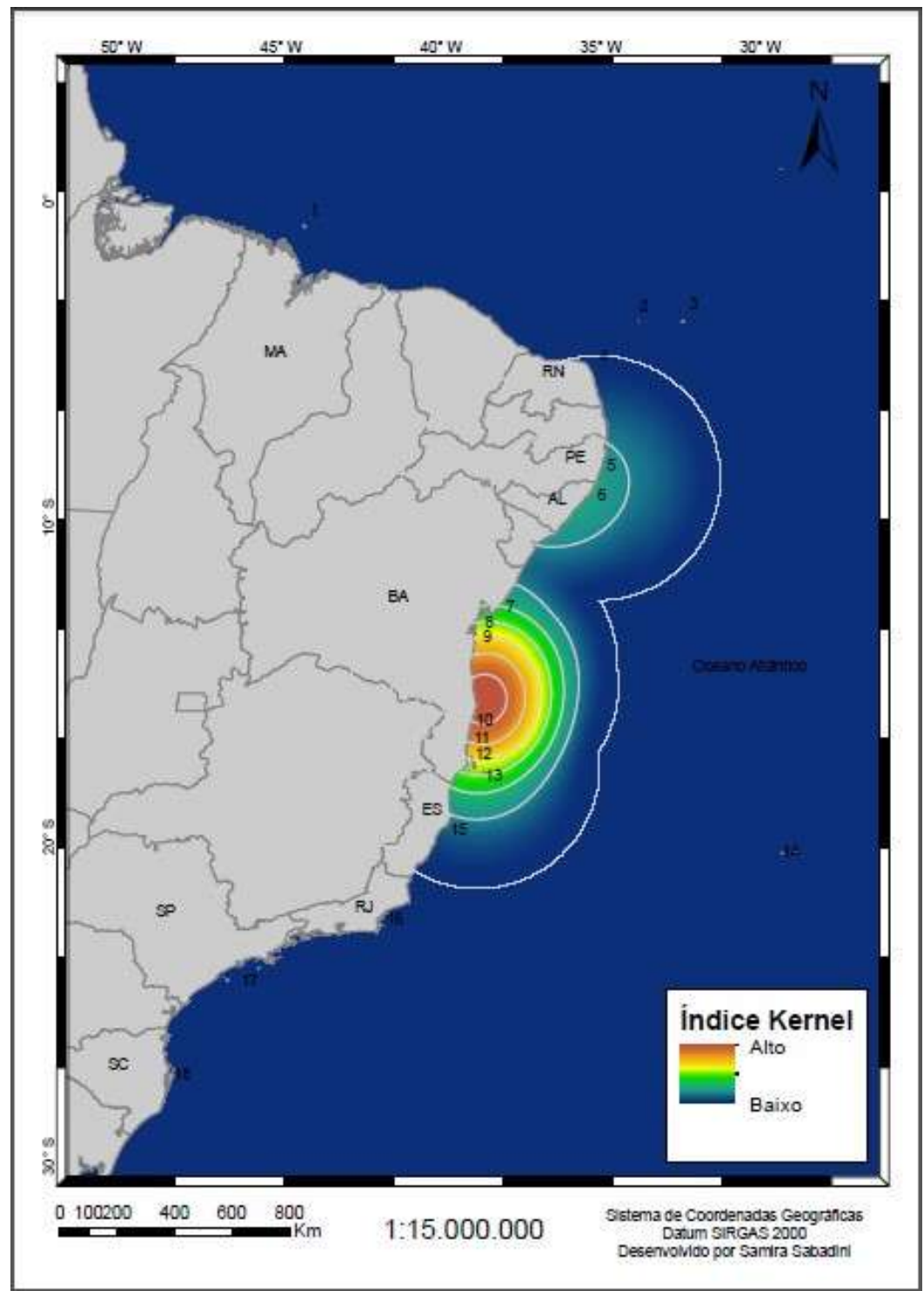

Figura 4: Áreas de endemismo de corais escleractíneos e hidrocorais na costa brasileira. 1. Parcel Manuel Luís; 2. Atol das Rocas; 3. Arquipélago de Fernando de Noronha; 4. Maracajaú; 5. Tamandaré; 6. Maragogi; 7. Litoral Norte da Bahia; 8. Baía de Todos os Santos; 9. Ilhas de Tinharé, Boipeba e Camamu; 10. Cabrália; 11. Itacolomis; 12. Timbebas; 13. Abrolhos; 14. Ilha de Trindade; 15. Costa do Espírito Santo; 16. Costa do Rio de Janeiro; 17. Costa de São Paulo; 18. Costa de Santa Catarina.

A área de endemismo adjacente aos Estados do Rio Grande do Norte, Pernambuco e Alagoas apresentou baixo Índice Kernel com maior intensidade na região da Costa dos Corais (5 e 6). Na costa do Estado da Bahia foi identificada a mais intensa área de endemismo com alto Índice Kernel na região de Cabrália (10) e Itacolomis (11). As ilhas oceânicas (3 e 14), Atol das Rocas (2) e a costa dos Estados localizados na zona de desaparecimento dos corais (16, 17 e 18) não apresentaram relevante Índice Kernel e, portanto, são regiões de baixo endemismo de espécies de corais hermatípicos. 
Este cenário é corroborado pelas pesquisas de vários autores (LEÃO, 1999, p. 345; FERREIRA et al, 2006, p. 1012; KIKUCHI et al, 2010, p. 5) que relacionam a maior diversidade de espécies de corais e hidrocorais endêmicos no Oceano Atlântico Sul Ocidental à região de Abrolhos (BA).

Visando um melhor nível de detalhes a respeito das áreas de endemismo de corais

na costa do Estado da Bahia, foi aplicado o método GIE em escala regional. Também foram identificadas duas áreas de endemismo, sendo a de maior intensidade novamente localizada em Cabrália (10). Na Figura 5 são apresentadas as áreas de endemismo de espécies de corais hermatípicos na costa do Estado da Bahia.

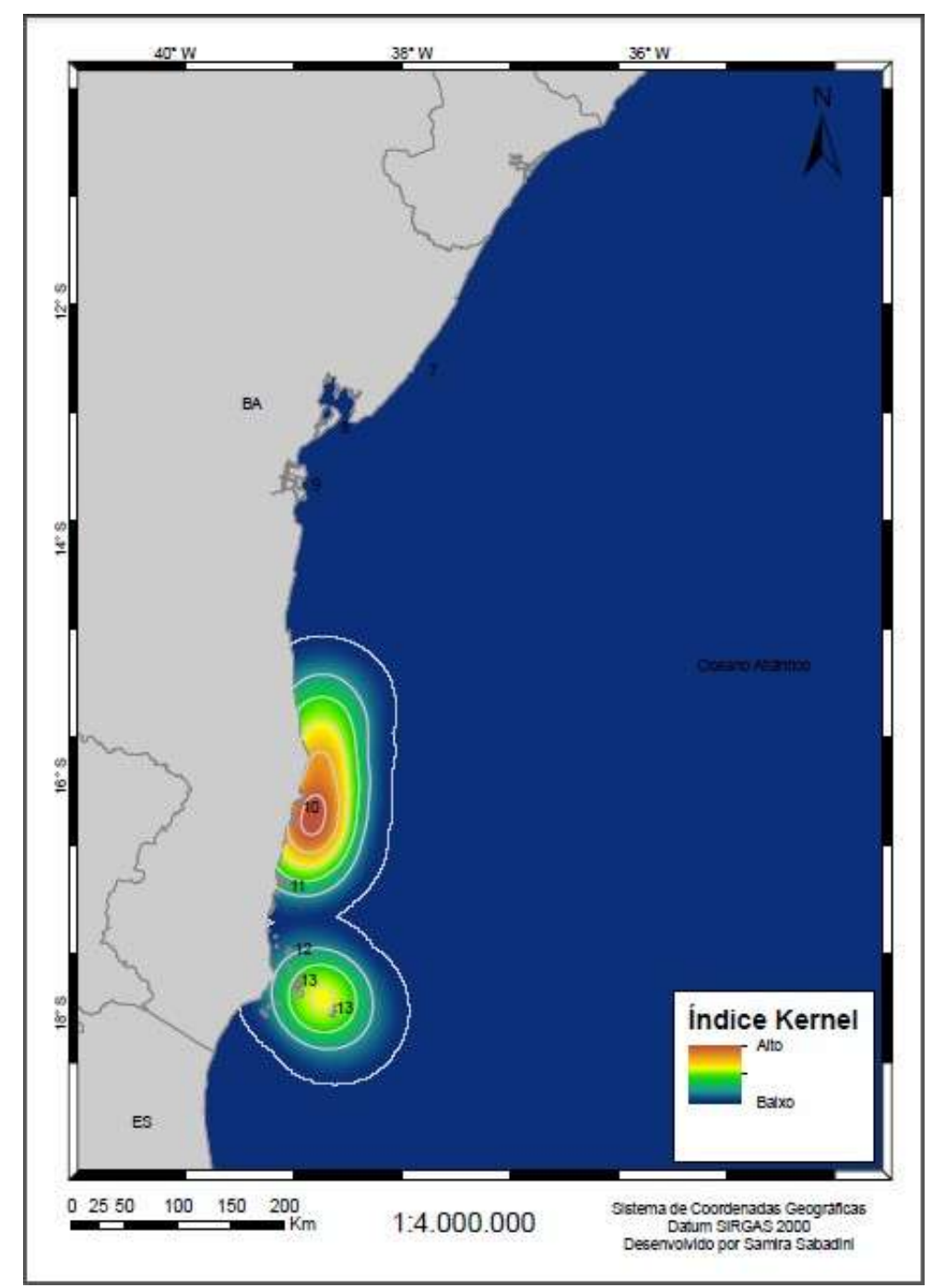

Figura 5: Áreas de endemismo de corais escleractíneos e hidrocorais na costa da Bahia. 7. Litoral Norte da Bahia; 8. Baía de Todos os Santos; 9. Ilhas de Tinharé, Boipeba e Camamu; 10. Cabrália; 11.

Itacolomis; 12. Timbebas; 13. Abrolhos. 
As regiões do litoral norte da Bahia (7), Baía de Todos os Santos (8), Ilhas de Tinharé e Boipeda e Baía de Camamu (9) apresentaram valores muito baixos de Índice Kernel. Os recifes de Itacolomis (11) e Timbebas (12) apresentaram valores baixos de índice Kernel, enquanto o Complexo Recifal de Abrolhos (13) apresentou valores intermediários de índice Kernel.

Apesar do maior número de espécies de corais hermatípicos endêmicos na região de Abrolhos, com tendência à diminuição da quantidade de espécies em direção ao norte da Bahia (LEÃO et al, 2010, p. 226), a região de Cabrália superou Abrolhos na intensidade de endemismo de espécies. Este resultado sugere que os centroides de distribuição de algumas espécies estão posicionados ao norte de Abrolhos. Esse cenário se deve à escassez de registros de espécies na zona de desaparecimento de corais (Sudeste e Sul do Brasil) e existência de recifes nos outros Estados do Nordeste do País.

$\mathrm{Na}$ costa brasileira há Unidades de Conservação de áreas recifais Marinhas de âmbito Federal, Estadual e Municipal visando à conservação da biodiversidade. Na Figura 6 são apresentadas as unidades federais de conservação de áreas recifais divididas em duas categorias: Proteção Integral e Uso Sustentável. 


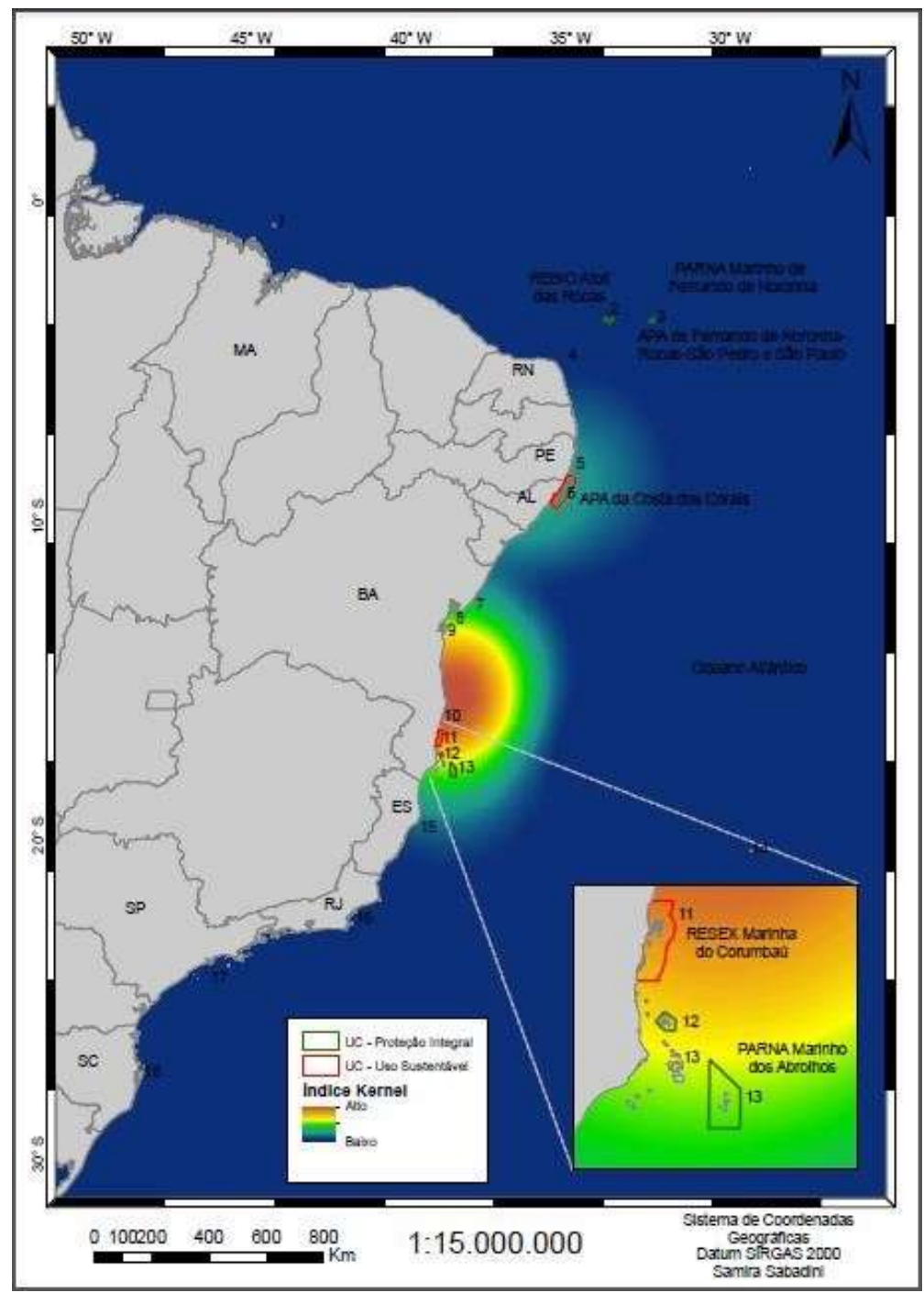

Figura 6: Unidades de Conservação Federais na costa brasileira.

As regiões de Atol das rocas (2) e Fernando de Noronha (3), que apresentam baixo endemismo de corais hermatípicos, estão bem protegidos pela Reserva Biológica do Atol das Rocas (RN) e pelo Parque Nacional Marinho de Fernando de Noronha (PE). Ambas são Unidades de Conservação Federais de Proteção Integral.

O Parcel de Manuel Luis (1), que também apresenta baixo endemismo de espécies, não é protegido por nenhuma Unidade de Conservação Federal. No entanto, é protegido dentro dos limites do Parque Estadual Marinho do Parcel do Manuel Luis que, assim como grande parte das unidades de conservação estaduais e municipais, possui grandes desafios em relação ao cumprimento dos objetivos de uma unidade de conservação.

Os Parrachos de maracajaú (4) também não apresentam relevante endemismo de corais hermatípicos. Esses recifes encontram-se menos protegidos que o Parcel de Manuel Luis, pois estão inseridos na Área de Proteção Ambiental Estadual dos Recifes de Corais (APARC) que é 
uma Unidade de conservação de Uso Sustentável. Segundo (LOPES et al, 2014), o principal problema existente na APARC é a falta de planejamento do turismo que afeta os recifes de corais durante a ancoragem dos barcos de turismo e pelo elevado número de visitantes. Prates (2003, p. 108) ainda cita a falta de infraestrutura da APARC.

Os recifes de Tamandaré (5) e Maragogi (6), localizados na área de endemismo menos expressiva, encontram-se protegidos pela Área de Proteção Ambiental Costa dos Corais (PE/AL). Recentemente, a APA Costa dos Corais, que é a maior Unidade de Conservação Marinha na esfera Federal, passou a receber mais proteção com a criação de duas Zonas de Preservação da Vida Marinha onde não são permitidas ações antrópicas (MMA, acesso em 20 nov. 2015).

Dentre os recifes da costa da Bahia, aqueles localizados no Litoral Norte (7) apresentaram os menores índices Kernel. Esses recifes são protegidos a nível estadual por meio da Área de Proteção Ambiental do Litoral Norte que apresenta como principais desafios os impactos do turismo e a ocupação desordenada do solo (INEMA, acesso em 20 nov. 2015).

Os recifes da baía de Todos os Santos (8) apresentam intensidade intermediária de endemismo, assim como o Complexo Recifal de Abrolhos (13). Ambos são protegidos por unidades de conservação estaduais de Uso Sustentável (Área de Proteção Ambiental da Baía de Todos os Santos e Área de Proteção Ambiental Ponta da Baleia/Abrolhos). O Complexo recifal de Abrolhos também é protegido, embora não em sua totalidade, pelo Parque Nacional Marinho de Abrolhos (BA).

A região das Ilhas de Tinharé e Boipeda e Baía de Camamu (9), assim como os recifes de Timbebas (12), apresentam considerável índice Kernel. Com exceção de Timbebas, as demais áreas são protegidas apenas por Unidades de Conservação Marinhas Estaduais de Uso Sustentável (Área de Proteção Ambiental Tinharé-Boipeba e Área de Proteção Ambiental da Baía de Camamu). Já os recifes de Timbebas, inseridos dentro dos limites do Complexo Recifal de Abrolhos, estão protegidos pelo Parque Nacional Marinho de Abrolhos.

A região de Cabrália (10), que apresentou o mais alto índice Kernel, não apresenta Unidades de Conservação Federais. No entanto, é protegida por duas Unidades de Conservação de Proteção Integral: O Parque Municipal Marinho da Coroa Alta e o Parque Municipal Marinho do Recife de Fora. Segundo Prates (2003, p. 141), o Ministério Público já cobrou explicações da Prefeitura de Porto Seguro a respeito da ineficiência da administração do Parque Municipal Marinho do Recife de Fora. 
Os recifes de Itacolomis (11) apresentam o segundo maior índice Kernel e, portanto, é uma importante área de sindemia de corais hermatípicos. A Reserva Extrativista Marinha de Corumbau protege esses recifes.

As demais regiões $(14,15,16,17$ e 18) não possuem Unidades de Conservação de áreas recifais e não apresentaram índices Kernel consideráveis, o que é compatível com a zona de empobrecimento de recifes.

\section{6- CONSIDERAÇÕES FINAIS}

Embora seja um ecossistema extremamente sensível e importante devido aos serviços ecossistêmicos prestados à sociedade, as Unidades de Conservação de áreas recifais no Brasil ainda são insuficientes para proteger o extenso território marinho. Atualmente, apenas 1,57\% desse território é protegido, ainda que não tenham sido alcançados todos os objetivos que foram propostos nos decretos de criação dessas unidades.

Diversos autores destacam a importância de Abrolhos por representar um hotspot de biodiversidade de corais endêmicos no Brasil. No entanto, o método GIE delimitou duas áreas de endemismo de corais, constituindo a região de Cabrália um hotspot de endemismo. É válido ressaltar, que Cabrália recebe proteção questionável do Parque Municipal Marinho do Recife de Fora. Diante disso, é fundamental ampliar as pesquisas e fiscalizar a eficiência da Unidade de Conservação.

Conforme relatado por Prates (2003), a criação de unidades de conservação não garante a proteção dos recifes, já que muitas não possuem condições básicas de funcionamento ou plano de manejo. Logo, é imprescindível o investimento em infraestrutura, fiscalização e conscientização da sociedade para que as unidades de conservação tornem-se, de fato, instrumentos de preservação das áreas recifais.

\section{AGRADECIMENTOS}

A autora agrade a CAPES pela bolsa de mestrado, aos professores da disciplina e a todos que de alguma forma contribuíram para o desenvolvimento deste trabalho. 


\section{REFERÊNCIAS BIBLIOGRÁFICAS}

CASTRO, C. B. Recifes de coral. 2000. Disponível em: http://www.icmbio.gov.br/parnaabrolhos/images/stories/downloads/Clovis 2000.pdf. Acesso em: 17 nov 2015.

CAVALCANTE, F. R. B.; BORGES, S. C. A.; SANTANA, E. F. C.; AMARAL, F. D. Checklist e abundância dos cnidários nos ambientes recifais de Maragogi, Alagoas. Tropical Oceanography, Recife, v. 42, n. 2, p. 208-217, 2014.

ECHEVERRíA, C. A.; PIRES, D. O.; MEDEIROS, M. S.; CASTRO, C. B. Cnidarians of the Atol das Rocas, Brazil, Proc. No. 8 of theInternational Coral ReefSymposium, Panama, 1: 443446. 1997.

FERREIRA, B. P.; MAIDA, M.; CASTRO, C. B.; PIRES, D. O.; D’AMICO, T. M.; PRATES, A. P. L.; MARX, D. The Status of Coral Reefs in Brazil. Proceedings. No. 10 of theInternational Coral ReefSymposium, Japan, 1011-1015. 2006.

INSTITUTO BRASILEIRO DE GEOGRAFIA E ESTATÍSTICA (IBGE/DGC). Base Cartográfica Contínua, ao milionésimo - BCIM: versão 4.0. Rio de Janeiro, 2014. Disponível em: <ftp://geoftp.ibge.gov.br/mapeamento_sistematico/base_continua_ao_milionesimo/BCIM_V4 / >. Acesso em: 04 nov. 2015.

INSTITUTO DO MEIO AMBIENTE E RECURSOS HÍDRICOS (INEMA). APA Litoral Norte do Estado da Bahia. Disponível em: <http://www.inema.ba.gov.br/gestao-2/unidadesdeconservacao/apa/apa-litoral-norte-do-estado-da-bahia/ >. Acesso em: 20 nov. 2015.

KIKUCHI, R. K. P.; LEÃO, Z. M. A. N.; OLIVEIRA, M. D. M.; DUTRA, L. X. C.; CRUZ, I. C. Branqueamento de corais nos recifes da Bahia associado aos efeitos do El Niño 2003. In: Congresso Sobre Planejamento e Gestão Das Zonas Costeiras Dos Países De Expressão Portuguesa, 2 / Congresso Da Associação Brasileira De Estudos Do Quaternário - ABEQUA, 9 / Congresso Do Quaternário De Países De Língua Portuguesa, 2, 2003, Recife. Anais. 2003.

KIKUCHI, R. K. P.; LEÃO, Z. M. A. N; OLIVEIRA, M. D. M. Conservation status and spatialpatterns of AGRRA vitalityindices in SouthwesternAtlanticReefs. Revista BiolTrop, San José, v. 58, suppl 1, p. 1-31, 2010.

LABOREL, J. L. Madreporaires et hydrocoralliairesrecifauxdescôtesbrésiliennes. Systematique, ecologie, repartitionverticale et geographie. Ann. Inst. Oceanogr. Paris 47: 171-229.

LEÃO, Z. M. A. N. Abrolhos - O complexo recifal mais extenso do Oceano Atlântico Sul. In: SCHOBBENHAUS, C.; CAMPOS, D. A.; QUEIROZ, E. T.; WINGE, M.; BERBERTBORN, M. (Eds.), SIGEP, Sítios Geológicos e Paleontológicos do Brasil, 1999, p. 345-359.

LEÃO, Z. M. A. N.; KIKUCHI, R. K. P. TESTA, V. Corals and coral reefs of Brasil. In: CORTES, J. (Ed.), Elsevier Science, Latin American Coral Reefs, 2003, p. 9-52. 
LEÃO, Z. M. A. N.; KIKUCHI, R. K. P.; OLIVEIRA, M. D. M.; VASCONCELLOS, V. Status of EasternBrazilian coral reefs in time of climatechanges. Pan-AmericanJournal of Aquatic Science, v. 5, n. 2, p. 224-235, 2010.

LOPES, R. M. R.; SOARES, I. A.; ARAÚJO, J. S. S. de. Área de Proteção Ambiental dos Recifes de Corais - Área dos Parrachos de Maracajaú/RN: desafios para o uso sustentável. Caminhos de Geografia, Uberlândia, v. 15, n. 51, p. 216-236, 2014.

MINISTÉRIO DO MEIO AMBIENTE (MMA). Recifes de Coral. Disponível em: <http://www.mma.gov.br/biodiversidade/biodiversidade-aquatica/zona-costeiraemarinha/recifes-de-coral>. Acesso em: 03 nov. 2015.

MINISTÉRIO DO MEIO AMBIENTE (MMA). Proposição de Unidades de Conservação na Região dos Abrolhos. 2012. Disponível em: $<$ http://www.icmbio.gov.br/portal/images/stories/oquefazemos/Propostas_UCs_Banco_Abrolhos_0512_novo2.pdf>. Acesso em: 04 nov 2015.

MINISTÉRIO DO MEIO AMBIENTE (MMA). Unidades de Conservação e Mosaicos. Disponível em: <http://www.mma.gov.br/biodiversidade/biodiversidadeaquatica/zonacosteira-e-marinha/unidades-de-conservacao-e-mosaicos $>$. Acesso em: 03 nov 2015.

MINISTÉRIO DO MEIO AMBIENTE (MMA). APA Costa dos Corais ganha mais proteção. Disponível em: < http://www.icmbio.gov.br/portal/comunicacao/noticias/4destaques/7166apa-costa-dos-corais-ganha-mais-protecao.html>. Acesso em: 20 nov 2015.

OLIVEIRA, U.; BRESCOVIT, A. D.; SANTOS, A. J. DelimitingAreas of EndemismthroughKernelInterpolation. PLoS ONE. v. 10, n. 1, p. 1-18, 2015.

PRATES, A. P. L. Recifes de Coral e Unidades de Conservação Costeiras e Marinhas no Brasil: uma análise da representatividade e eficiência na conservação da biodiversidade. 2003. 175 f. Tese (Doutorado em Ecologia). Universidade de Brasília, Brasília, 2003.

PINHEIRO, B. R. Recrutamento de corais no Recife da Ilha da Barra - Tamandaré / PE. 2006. 69 f. Dissertação (Mestrado em Ciências). Universidade Federal de Pernambuco, Recife, 2006.

SANTOS, C. L. A.; VITAL, H.; AMARO, V. E.; KIKUCHI, R. K. P. de. Mapeamento de recifes submersos na costa do Rio Grande do Norte, Ne Brasil: Macau a Maracajau. Revista Brasileira de Geofísica, São Paulo, v. 25. p. 27-36. 2007.

SERVIÇO GEOLÓGICO DO BRASIL (CPRM). Geobank. Disponível em: < http://www.cprm.gov.br/publique/cgi/cgilua.exe/sys/start.htm?infoid=3224\&sid=122>.

Acesso em: 29 out. 2015. 


\section{APÊNDICE 139}

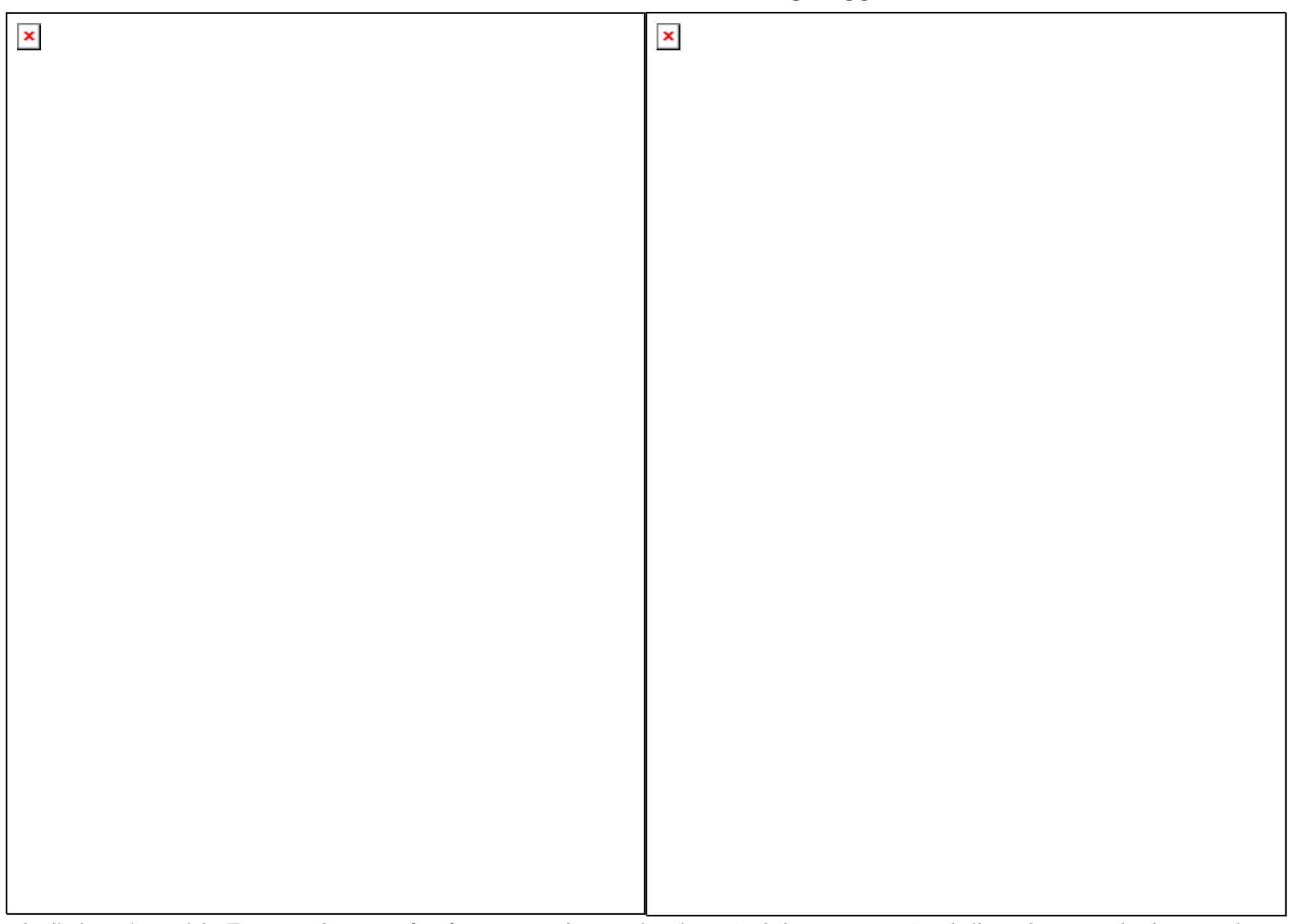

Distribuição da espécie Favia gravida na costa brasileira. 1. Parcel Manuel Luís; 2. Atol das Rocas; 3. Arquipélago de Fernando de Noronha; 4. Maracajaú; 5. Tamandaré; 6. Maragogi; 7. Litoral Norte da Bahia; 8. Baía de Todos os Santos; 9. Ilhas de Tinharé, Boipeba e Camamu; 10. Cabrália; 11. Itacolomis; 12. Timbebas; 13. Abrolhos; 14. Ilha de Trindade; 15. Costa do Espírito Santo; 16. Costa do Rio de Janeiro; 17. Costa de São Paulo; 18. Costa de Santa Catarina.

Cadernos do Leste

Artigos Científicos 


\section{APÊNDICE B}

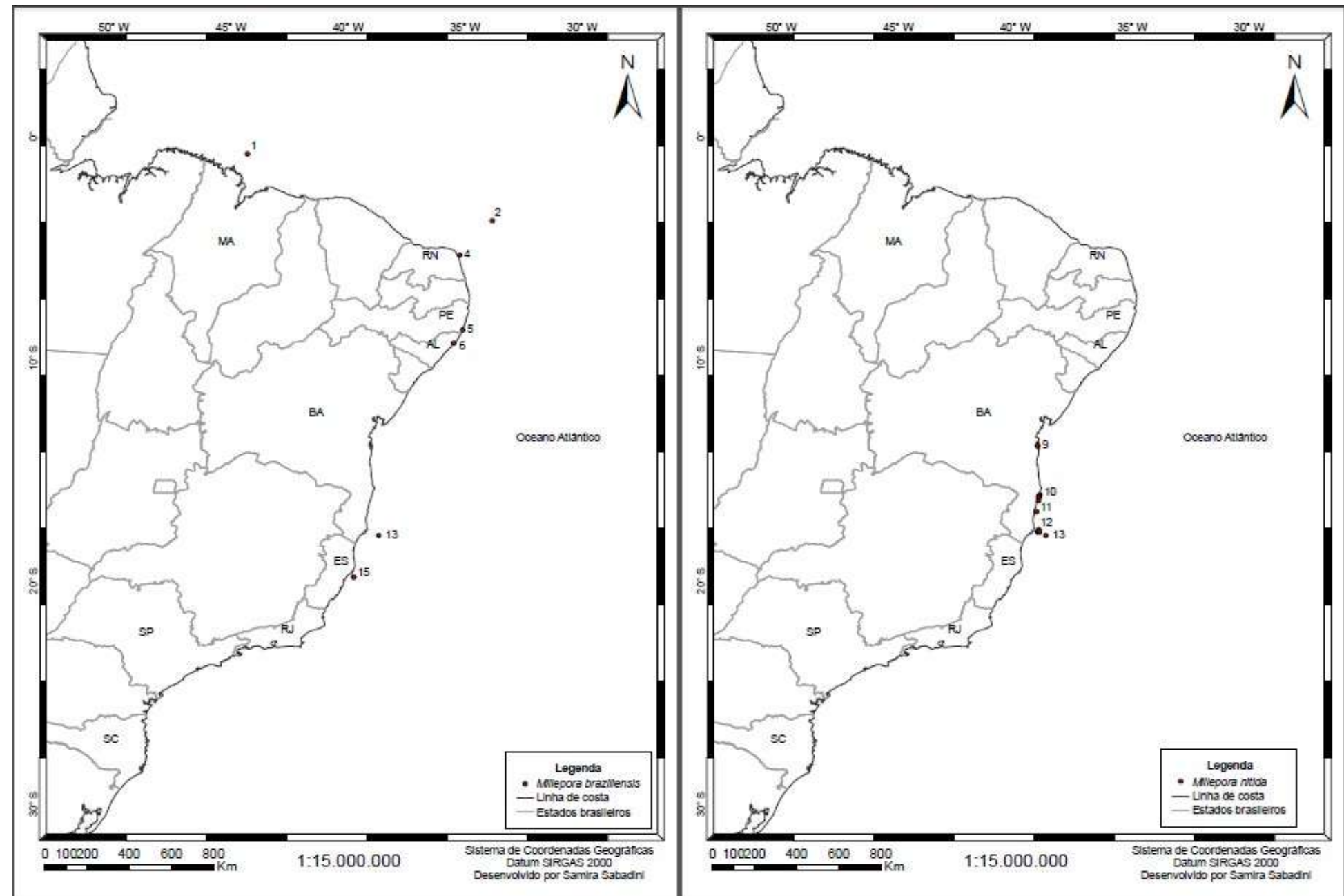

Distribuição da espécie Milleporabraziliensisna costa brasileira. 1. Parcel Manuel Luís; 2. Atol das Rocas; 3. Arquipélago de Fernando de Noronha; 4. Maracajaú; 5. Tamandaré; 6. Maragogi; 7. Litoral Norte da Bahia; 8. Baía de Todos os Santos; 9. Ilhas de Tinharé, Boipeba e Camamu; 10. Cabrália; 11. Itacolomis; 12. Timbebas; 13. Abrolhos; 14. Ilha de Trindade; 15. Costa do Espírito Santo; 16. Costa do Rio de Janeiro; 17. Costa de São Paulo; 18. Costa de Santa Catarina.

\section{Cadernos do Leste}


APÊNDICE C
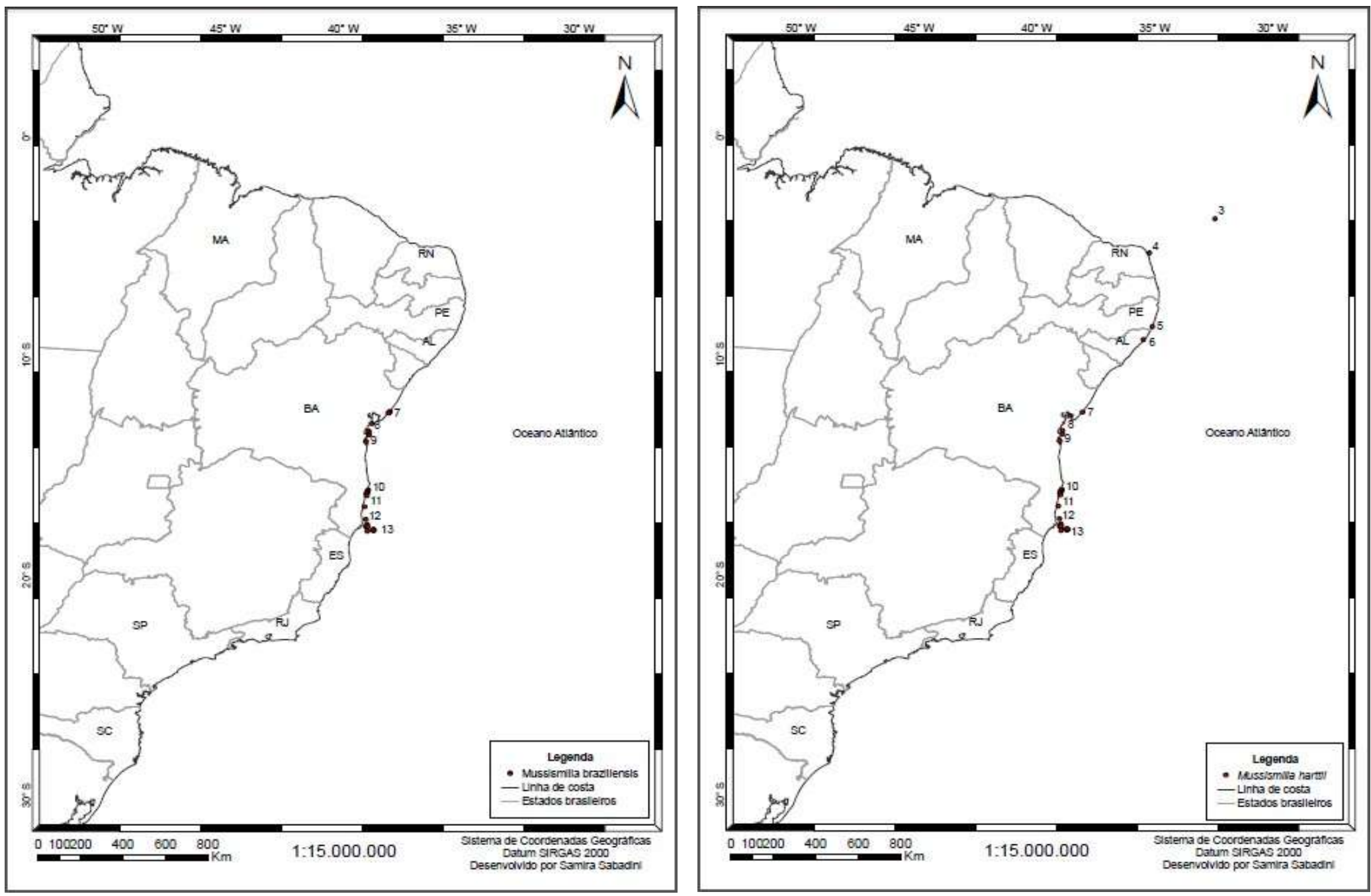
Distribuição da espécie Mussismiliabraz̨iliensisna costa brasileira. 1. Parcel Manuel Luís; 2. Atol das Rocas; 3. Arquipélago de Fernando de Noronha; 4. Maracajaú; 5. Tamandaré; 6. Maragogi; 7. Litoral Norte da Bahia; 8. Baía de Todos os Santos; 9. Ilhas de Tinharé, Boipeba e Camamu; 10. Cabrália; 11. Itacolomis; 12. Timbebas; 13. Abrolhos; 14. Ilha de Trindade; 15. Costa do Espírito Santo; 16. Costa do Rio de Janeiro; 17. Costa de São Paulo; 18. Costa de Santa Catarina.

APÊNDICE D

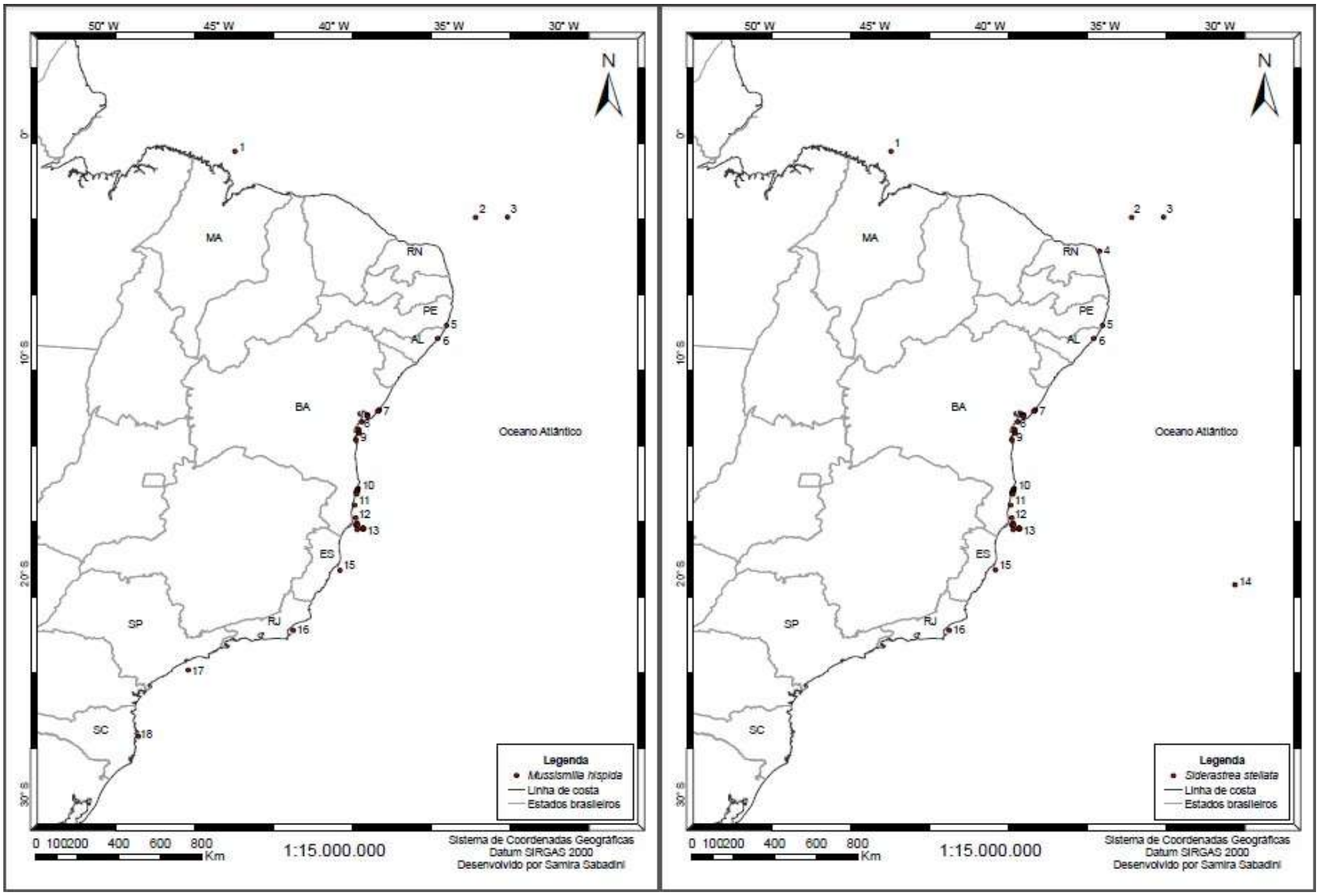

Cadernos do Leste

Artigos Científicos

Belo Horizonte, Jan.-Dez. Vol.16, n¹6, 2016 
Distribuição da espécie Mussismilia hispida na costa brasileira. 1. Parcel Manuel Luís; 2. Atol das Rocas; 3. Arquipélago de Fernando de Noronha; 4. Maracajaú; 5. Tamandaré; 6. Maragogi; 7. Litoral Norte da Bahia; 8. Baía de Todos os Santos; 9. Ilhas de Tinharé, Boipeba e Camamu; 10. Cabrália; 11. Itacolomis; 12. Timbebas; 13. Abrolhos; 14. Ilha de Trindade; 15. Costa do Espírito Santo; 16. Costa do Rio de Janeiro; 17. Costa de São Paulo; 18. Costa de Santa Catarina. 